Copyright (C 2006 IEEE. Reprinted from IEEE Sensors Journal, 2006; $6(6): 1473-1481$

This material is posted here with permission of the IEEE. Such permission of the IEEE does not in any way imply IEEE endorsement of any of the University of Adelaide's products or services. Internal or personal use of this material is permitted. However, permission to reprint/republish this material for advertising or promotional purposes or for creating new collective works for resale or redistribution must be obtained from the IEEE by writing to pubs-permissions@ieee.org.

By choosing to view this document, you agree to all provisions of the copyright laws protecting it. 


\title{
Using Smart Sensor Strings for Continuous Monitoring of Temperature Stratification in Large Water Bodies
}

\author{
Andrew J. Skinner and Martin F. Lambert
}

\begin{abstract}
A "smart" thermistor string for continuous longterm temperature profiling in large water bodies is described allowing highly matched yet low-cost spatial and temporal temperature measurements. The sensor uses the three-wire SDI-12 communications standard to enable a low-powered radio or data logger on supporting buoys to command measurements and retrieve high-resolution temperature data in digital form. Each "smart" temperature sensor integrates a thermistor element, measurement circuitry, power control, calibration coefficient storage, temperature computation, and data communications. Multiple addressable sensors at discrete vertical depths are deployed along a three-wire cable that provides power and allows data transfer at regular intervals. Circuit, manufacturing, and automated calibration techniques allow temperature measurements with a resolution of $\pm 0.003{ }^{\circ} \mathrm{C}$, and with intersensor matching of $\pm 0.006{ }^{\circ} \mathrm{C}$. The low cost of each sensor is achieved by using poor tolerance thermistor and circuit components in conjunction with a 15-bit charge-balance analog-to-digital converter. Sensor inaccuracies and temperature coefficients are corrected by a two-point calibration procedure made possible by a standard-curve generator within the sensor, based upon the method of finite differences. This two-point calibration process allows in-field sensor string calibration in stratified water bodies and provides a means to correct for long-term calibration drift without having to return the string to a laboratory.
\end{abstract}

Index Terms-Analog-to-digital converter (ADC), method of finite differences, SDI-12, smart sensor, stratification, temperature, thermistor strings.

\section{INTRODUCTION}

$\mathbf{M}$ EASUREMENT of thermal stratification in lakes and reservoirs can be a valuable tool in predicting changes in water quality. What is poorly understood about this measurement process is that sensor matching is more important than sensor accuracy, because it is the rate of temperature change with depth, not the temperature value itself, that is important.

Manuscript received November 5, 2005; revised June 6, 2006. This work was supported by Measurement Engineering Australia of Adelaide South Australia as part of an industrial Ph.D. program in association with the Department of Civil and Environmental Engineering at Adelaide University. The associate editor coordinating the review of this paper and approving it for publication was Dr. Usha Varshney.

A. J. Skinner is with the Measurement Engineering Australia Adelaide, South Australia, Australia (e-mail: andrew.skinner@mea.com.au).

M. F. Lambert is with the School of Civil and Environmental Engineering at Adelaide University, Adelaide, South Australia 5005, Australia (e-mail: mlambert@civeng.adelaide.edu.au).

Color versions of Figs. 2 and 4-7 are available at http://ieeexplore.ieee.org.

Digital Object Identifier 10.1109/JSEN.2006.881373
This is especially true when thermoclines are weak and sensors are close together.

Traditional temperature profiling for continuous long-term monitoring in natural water bodies has used multiple thermistor temperature sensors hanging from a structure housing a multichannel data logger [8]. This paper describes alternative "smart" temperature sensors developed for use in sensor strings used for monitoring vertical temperature stratification in large water bodies. These vertical sensor strings are designed for permanent monitoring of fine-scale temperature changes at meter length scales and for deployment in lines or grids.

A smart temperature sensor incorporates a basic thermistor sensor element, an analog-to-digital converter (ADC) to digitize the measurement, a microcontroller responsible for computations, control and communications, algorithms to convert the raw measurement into a calibrated temperature, software algorithms to correct for the temperature coefficients of the sensor's electronics, some means of updating and storing individual sensor calibration coefficients, and a means of communicating with and being addressed by data loggers, computers, or telemetry systems. Additionally, smart sensors have the ability to be calibrated automatically under the control of a computer that runs them through a calibration process against a standard and against each other, and where calibration coefficients for individual sensors are computed and written back into the sensors without human intervention. These techniques, once developed, allow production of lower-cost high-quality sensors with temperature matching better than $\pm 0.01{ }^{\circ} \mathrm{C}$, an order of magnitude improvement over the best factory calibrated devices.

\section{SEnsor Design}

\section{A. Temperature Sensing}

Negative temperature coefficient (NTC) thermistor temperature sensors offer the lowest cost, highest resolution, and easiest interfacing of a variety of temperature sensors. The thermistor used in this design is a Vishay BC Components 2322-633$83104100 \mathrm{k} \Omega \pm 10 \%$ at $25^{\circ} \mathrm{C}$ bead-in-glass thermistor costing less than $\$ 1$ and mounted directly onto the sensor printed circuit board (PCB) along with the three semiconductor components used in the sensor. Its resistance varies from about $333 \mathrm{k} \Omega$ at $0{ }^{\circ} \mathrm{C}$ to about $35 \mathrm{k} \Omega$ at $50{ }^{\circ} \mathrm{C}$. In this type of "point-match" thermistor, the resistance uncertainties become even larger at 
temperatures above and below the specified reference temperature of $25{ }^{\circ} \mathrm{C}$, due to the uncertainty in the material curve. A more expensive type of thermistor, known as a "curve matched" or "interchangeable" thermistor, is normally defined by a certain accuracy over a range, typically $\pm 0.2{ }^{\circ} \mathrm{C}$ (and at best, $\pm 0.1{ }^{\circ} \mathrm{C}$ ) from $0{ }^{\circ} \mathrm{C}$ to $70{ }^{\circ} \mathrm{C}$. By comparison, this thermistor's uncorrected temperature error has an uncertainty of $\pm 2.5^{\circ} \mathrm{C}$ due to the $\pm 10 \%$ resistance uncertainty at $100 \mathrm{k} \Omega$, but this can be reduced to $\pm 0.01{ }^{\circ} \mathrm{C}$ over the range $0{ }^{\circ} \mathrm{C}$ to $50{ }^{\circ} \mathrm{C}$ using the method to be described.

The Steinhart-Hart equation [10] for converting thermistor resistance $R_{\mathrm{T}}$ to temperature $T$ is an inverse third-order logarithmic polynomial having device-dependent coefficients $m_{1}$, $m_{2}$, and $m_{3}$. The equation is

$$
T(\mathrm{~K})=\frac{1}{m_{1}+m_{2} \ln \left(R_{\mathrm{T}}\right)+m_{3}\left(\ln \left(R_{\mathrm{T}}\right)\right)^{3}}
$$

For sensing in the narrow range of water temperatures found in natural water bodies $\left(-5{ }^{\circ} \mathrm{C}\right.$ to $\left.45{ }^{\circ} \mathrm{C}\right)$, this equation can be simplified by dropping the third-order term $m_{3}\left(\ln \left(R_{\mathrm{T}}\right)\right)^{3}$ and rearranging the equation to form (2)

$$
T\left({ }^{\circ} \mathrm{C}\right)=\frac{a}{\ln \left(R_{\mathrm{T}}\right)+b}-c
$$

where $a, b$, and $c$ are coefficients determined during calibration. The coefficient $c$ includes the conversion offset between Kelvin and Celsius temperature $\left(\mathrm{K}={ }^{\circ} \mathrm{C}+273.15\right)$. Where (1) fits data to $\pm 0.0001{ }^{\circ} \mathrm{C}$ over the temperature range of $-5{ }^{\circ} \mathrm{C}$ to $45^{\circ} \mathrm{C}$, (2) has an uncertainty of $\pm 0.002^{\circ} \mathrm{C}$.

This simplification allows the computation to fit into the 2000-instruction program space of the low-cost PIC16F628 microcontroller used at the heart of this sensor.

\section{B. Circuit Operation}

The circuit topology chosen for the ADC is a form of a synchronous voltage-to-frequency converter (SVFC) circuit based loosely on the design of the analog devices part number AD652 [1]. A simple op-amp and comparator (LM392N) and linear micropower voltage regulator (LP2951) are used in conjunction with the CMOS PIC16F628 microcontroller to form a 15-bit ADC. The raw output of the ADC is used by the microcontroller to compute a temperature value to a precision of about $0.003{ }^{\circ} \mathrm{C}$ over the temperature range $-5{ }^{\circ} \mathrm{C}$ to $45^{\circ} \mathrm{C}$.

Timing, logic, and switching functions are implemented in the microcontroller. The same microcontroller processes measurement and communication commands on the one-signalwire SDI-12 interface bus, which is a de-facto industry-standard data interface [9] for use with low-power environmental instruments and data loggers. It allows concurrent measurements from all sensors simultaneously while they are connected together on the one-wire data bus, as each sensor is capable of listening for addresses and instructions while in a highimpedance state. Only one sensor (or the data logger) can talk at any one time. The SDI-12 standard makes provision for powering sensors on the same three-wire cable via a $12-\mathrm{V}$ dc power connection.

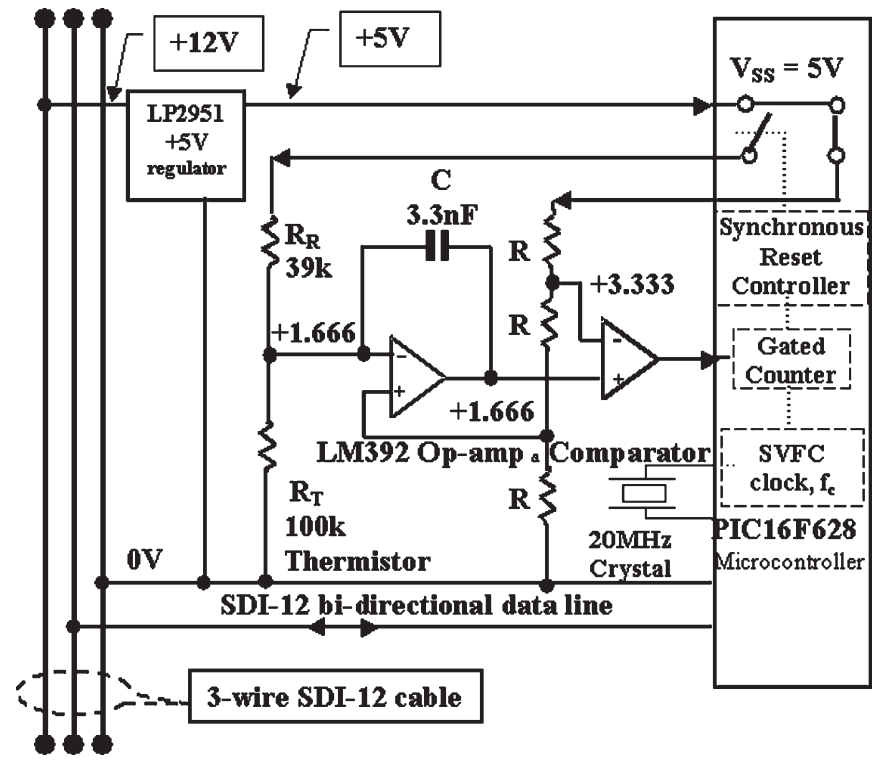

Fig. 1. Simplified sensor circuit diagram. The integration amplifier is on the left and the comparator on the right. Note that each sensor is essentially connected permanently across the three-wire SDI-12 bus that passes physically through each sensor down the whole length of the sensor string.

The temperature sensor of Fig. 1 can be thought of as a switched Wheatstone bridge circuit whose output feeds directly into an integrator rather than into an (expensive) instrumentation amplifier. The active left hand half of the bridge consists of the switched reset resistor $R_{\mathrm{R}}$ and the temperature-sensing thermistor $R_{\mathrm{T}}$. The right-hand reference side of the bridge generates the set points to the integrator and the comparator that make up the charge-balanced ADC. The two inputs of the op-amp are connected to opposite sides of the bridge.

The noninverting input of the integrator is fixed at a nominal voltage equal to $1 / 3$ of the supply voltage $V_{\mathrm{SS}}$ (nominally $+5.0 \mathrm{~V})$. This fixed voltage is effectively applied across the thermistor so that changes in thermistor resistance due to temperature changes alter the current through it. This current is supplied by the output stage of the integrator. The integrator output voltage is also connected to the noninverting input of the comparator, whose inverting reference input is fixed at a nominal voltage equal to $2 / 3$ of the supply voltage $V_{\text {SS }}$. The inverting input of the integrator is connected to ground via the thermistor. These mid-range threshold values of the integrator reference voltage at $1.666 \mathrm{~V}$ and the comparator reference voltage at $3.333 \mathrm{~V}$ allow the output of the integrator to swing smoothly midway between ground and the supply voltage without entering the nonlinear output regions of the amplifier near $0 \mathrm{~V}$ and $5 \mathrm{~V}$.

The very high resistance value of the thermistor (nominally $100 \mathrm{k} \Omega$ at $25^{\circ} \mathrm{C}$ ) prevents self-heating errors in the thermistor due to excessive power dissipation. For example, if the dissipation factor of the thermistor is $8 \mathrm{~mW} /{ }^{\circ} \mathrm{C}$ [13] inside the encapsulant, then a power dissipation caused by an applied voltage of $1.666 \mathrm{~V}$ across $100 \mathrm{k} \Omega$ would be $27.75 \mu \mathrm{W}$, which would generate a self-heating temperature rise error of $0.0035{ }^{\circ} \mathrm{C}$ at $25{ }^{\circ} \mathrm{C}$. The microcontroller and voltage regulator within the sensor dissipate 12 and $17 \mathrm{~mW}$ of power, respectively, for 
a period of $10 \mathrm{~s}$ while the measurement is in progress. The thermistor is located over 50-mm away from these components, while being only 10-mm away from the water. However, while this self-heating effect within the sensor compromises sensor accuracy, it does not affect intersensor matching, as all sensors have identical on-board heat sources.

The action of the integrator is to linearly ramp up its output voltage $V$ in order to drive a current equal to $C \cdot(d V / d t)$ through the integrating capacitor $C$ and to ground through the thermistor. The op-amp sets the feedback current so that the voltage across the thermistor is kept equal to the reference voltage of the integrator. The magnitude of this current and therefore the slope of the ramping output voltage is set by the thermistor resistance, and hence by the temperature. Therefore, the time taken for the output voltage of the integrator to reach the threshold voltage of the comparator is a function of temperature, and ultimately sets the pulse output rate of the ADC's comparator.

Once the output voltage of the integrator crosses the reference voltage threshold of the comparator, the comparator output switches high. This does not however immediately set a capacitor-reset operation into action in a SVFC. Instead, the output state of the comparator is sampled only on the falling edge of the crystal-locked internal SVFC clock generated in software within the microcontroller. If the comparator output state is detected to be high at this point, then the reset action is initiated on the next rising edge of the SVFC clock, and continues for a full (and very precise) clock period. This then strips a known amount of charge from the capacitor, resetting it, and turning OFF the output of the comparator.

The reset current is provided by switching the reset resistor $R_{\mathrm{R}}$ from a high-impedance state to $V_{\mathrm{SS}}(5 \mathrm{~V})$, causing current to flow through the capacitor to be sunk to ground by the op-amp output stage of the integrator, as well as through the thermistor to ground. The magnitude of the reset current is equal to $(5-1.666) V / R_{\mathrm{R}}=85 \mu \mathrm{A}$ and is at least equal to the current through the thermistor at the warmest operational temperature. That is, $R_{\mathrm{R}}$ has been set to $39 \mathrm{k} \Omega$, which is the resistance of the thermistor at about $47{ }^{\circ} \mathrm{C}$ - the maximum expected temperature of a sensor on a reservoir off-take tower exposed to direct sunshine if the water column has dropped below the top sensors in a smart sensor string.

During the reset cycle, the op-amp once again seeks to hold its two inputs at the same potential, equal to the integrator reference voltage. To do this, it must ramp the integrator output voltage $V$ down in order to sink the surplus reset current. The output of the integrator is therefore a temperature-dependent sawtooth waveform as shown in the top trace of Fig. 2.

The sensor's microcontroller keeps track of the number of reset pulses (as a variable called count) issued in a fixed gating period $T_{G}$ equal to 65536 (or $2^{16}$ ) SVFC clock cycles. The maximum number of reset pulses (bottom trace, Fig. 2) that can be generated is equal to half the number of SVFC clock periods, because the reset pulse must be turned off to allow sampling of the comparator output state during the next clock cycle. This limits the maximum resolution of the sensor to 1 count in 32768 counts; this is then a 15-bit ADC. Higher resolution and therefore greater temperature precision can be gained simply

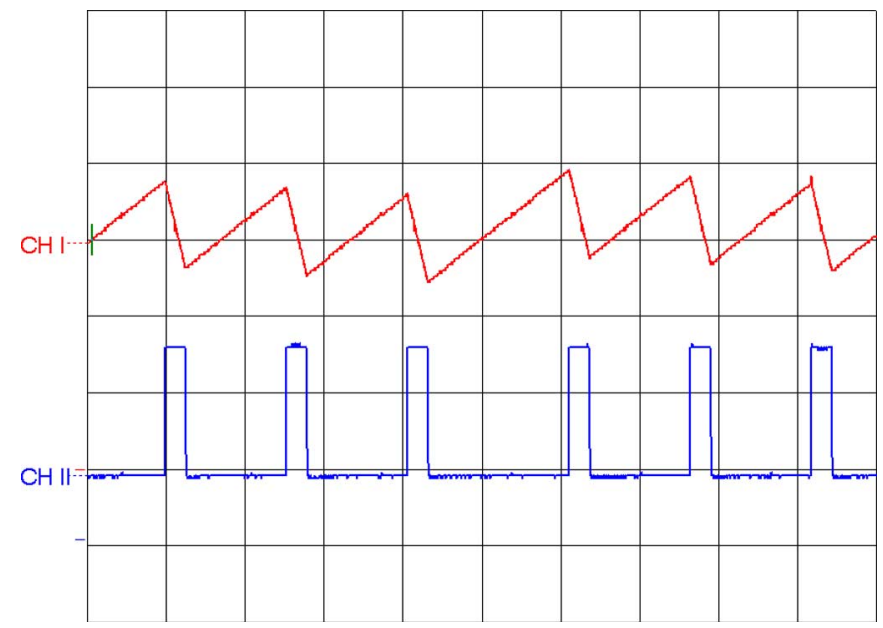

Fig. 2. Voltage waveforms within the $\mathrm{ADC}$ of the temperature sensor. $\mathrm{CH}$ (1 V/Div dc) is the sawtooth waveform at the output of the integrator; voltage rises during integration as the integration capacitor charges and falls during reset as this capacitor discharges. $\mathrm{CH}$ II (2 V/Div dc) shows the reset pulse (top of $R_{\mathrm{R}}$ ) switching on to discharge the integrator capacitor. It is at $1.666 \mathrm{~V}$ during integration and at $5 \mathrm{~V}$ during reset. Note that the reset pulses are not triggered at a specific threshold voltage, but on the next rising clock edge after the threshold voltage is exceeded. Waveforms were captured using a Hameg Instruments HM1507-three digital analog oscilloscope with a time base of $200 \mu$ s per division.

by increasing the gating period $T_{G}$, but at the cost of increased power consumption.

The count value is plugged into the temperature conversion algorithm in conjunction with the sensor-specific calibration coefficients in order to calculate true temperature.

\section{ADC Transfer Function}

It can be shown that during all the integration cycles throughout a gating period $T_{G}$ that the total charge $Q_{I}$ in coulombs added to the integration capacitor is

$$
-Q_{I}=T_{G} \times \frac{(65536-\text { count })}{65536} \times \frac{V_{\mathrm{SS}}}{3 R_{\mathrm{T}}}
$$

where $T_{G}$ is the gating period of the $\mathrm{ADC}$ equal to 65536 cycles of the basic SVFC frequency $f_{\mathrm{C}}$ generated internally by the sensor's microcontroller (Fig. 1), $V_{\mathrm{SS}}$ is the $+5 \mathrm{~V}$ supply voltage, $R_{\mathrm{T}}$ is the resistance of the thermistor, and count is the number of times the integration capacitor is reset (or partially discharged) during the gating period. The negative value of charge in (3) is simply a convention based upon charge flow being positive if toward the output of the integrator op-amp.

It can be also be shown that during all the reset cycles throughout the same gating period $T_{G}$ that the total charge $Q_{R}$ in coulombs stripped off the integration capacitor is

$$
Q_{R}=T_{G} \times \frac{\text { count }}{65536} \times\left(\frac{2 V_{\mathrm{SS}}}{3 R_{\mathrm{R}}}-\frac{1 V_{\mathrm{SS}}}{3 R_{\mathrm{T}}}\right) .
$$

For charge balance

$$
Q_{R}+Q_{I}=0
$$




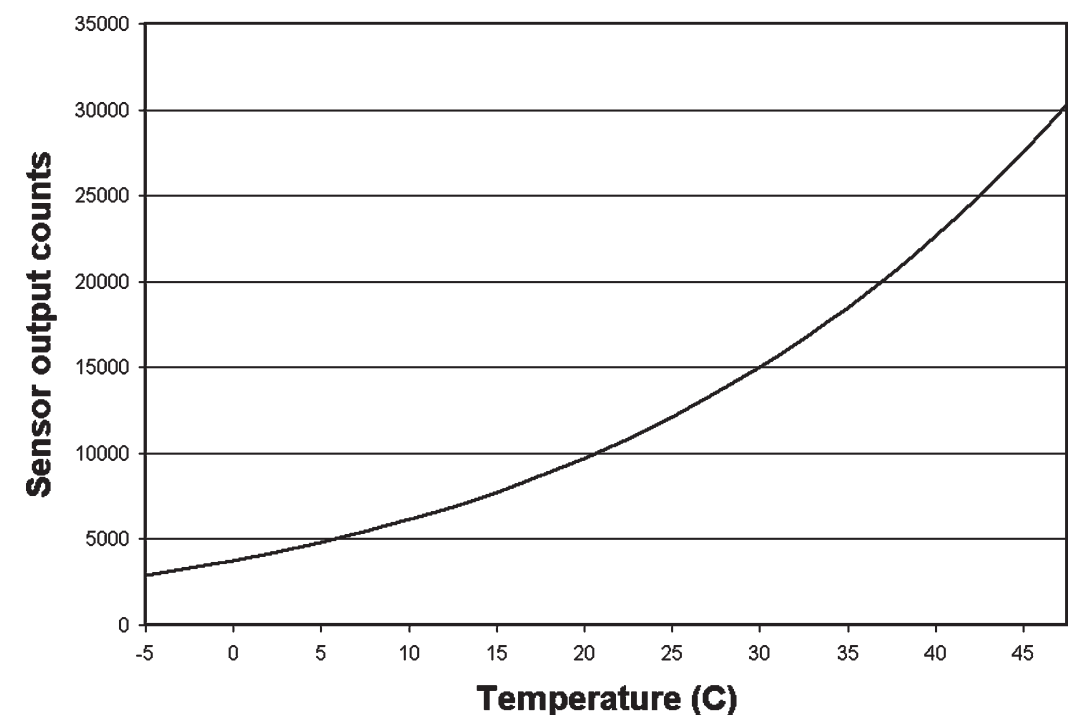

Fig. 3. "Count versus temperature" transfer function of the "standard" temperature sensor.

Therefore, by substituting terms from (3) and (4) into (5), an expression for the thermistor resistance is obtained

$$
R_{\mathrm{T}}=\frac{32768}{\text { count }} \times R_{\mathrm{R}}
$$

The unknown thermistor resistance $R_{\mathrm{T}}$ can be seen to be related quite simply to the known fixed resistance $R_{\mathrm{R}}$ by the inverse of the ADC output value count. The output count can be seen to be independent of the supply voltage $V_{\mathrm{SS}}$, the integrator capacitor value $C$, the clock frequency $f_{\mathrm{C}}$, and the gating period $T_{G}$. It is still dependent upon the temperature coefficient of the reset resistor $R_{\mathrm{R}}$.

The value of $R_{\mathrm{R}}$ in the design is set to the resistance value $R_{\mathrm{T} \max }$ of the thermistor at the warmest expected temperature; cooler temperatures increase the thermistor resistance and count falls from a maximum of 32768. Fig. 3 shows the count rate rising with temperature as described by (6). Note that (unlike standard thermistor sensors) the sensitivity of this sensor increases with temperature because of the inverse relationship between counts and thermistor resistance.

\section{Correcting for Temperature Drifts in Electronic Components}

The advantage of this temperature sensor design is that the circuit components and the measurement thermistor are in close proximity and immersed in the water body, and can therefore be considered to be at the same temperature. No separate measurement of circuit temperature is then needed to correct for the temperature coefficients of low-cost passive and active electronic components. Instead, these drifts and errors are picked up in the temperature conversion algorithm by the lumped temperature coefficient " $d$ " in

$$
R_{\mathrm{T}}=\frac{32768}{d \times(\text { temperature }-20) \times \text { count }+ \text { count }} \times R_{\mathrm{R}} .
$$

Rather than insert a temperature offset as well, the temperature is calculated with respect to a fixed temperature in the middle of the measurement range-in this case, at $20^{\circ} \mathrm{C}$.

The temperature of the thermistor can be found from its resistance via the limited temperature range Steinhart-Hart equation of (2). Substituting (7) into (2) gives

$$
T\left({ }^{\circ} \mathrm{C}\right)=\frac{a}{\ln \left(\frac{32768}{d \times\left(T_{\mathrm{c}}-20\right) \times \text { count }+ \text { count }} \times R_{\mathrm{R}}\right)+b}-c .
$$

$T_{\mathrm{c}}$ is a "correction temperature" made numerically equal to the bath temperature $T$ in the calibration curve-fitting process used to find calibration coefficients $a, b, c$, and $d$. The calibration coefficients for (8) were found from a 2530 point calibration of a single nominated "standard" sensor over the range $2{ }^{\circ} \mathrm{C}$ to $30{ }^{\circ} \mathrm{C}$, with an average deviation of $0.0012{ }^{\circ} \mathrm{C}$ and a maximum deviation for any observation of $0.0051^{\circ} \mathrm{C}$. If the sensor were to calculate temperature from (8) directly, an estimate for temperature $T$ would first be derived from a count value by forcing $T_{\mathrm{c}}$ to a value of $20{ }^{\circ} \mathrm{C}$. This estimate of $T$ would then be used as the new value for $T_{\mathrm{c}}$ and the process repeated at least fifteen times in a fixed-point iteration method that would produce a final accurate value of $T$ equal to $T_{\mathrm{c}}$.

\section{Computing Sensor Temperature by the "METHOD OF FINITE DifFERENCES"}

The basic algorithm for the sensor has been developed above as a first-order inverse logarithmic function incorporating a linear temperature correction term to adjust for passive component temperature coefficients. This function was computed by the microcontroller in early versions of the sensor but needed a significant amount of program memory to execute floating-point mathematics and to calculate natural logarithms, leaving little memory available for the communication functions required.

In low-cost sensor computations, where single-byte addition is often the only native arithmetic instruction available, an alternative approach using the method of finite differences has 
TABLE I

Eight Coefficients of a SEVEnth-Order Polynomial FitTing a Standard SEnsor's “Count Versus Temperature" Curve Over Two Temperature Ranges

\begin{tabular}{|r|l|l|}
\hline$\#$ & Coefficient value (low) & Coefficient value (high) \\
\hline $\mathrm{a}$ & -39123.457824960504 & -19478.797879792011 \\
\hline $\mathrm{b}$ & 20.383058346844802 & 7.0177896118638046 \\
\hline $\mathrm{c}$ & -0.0046910405108062814 & -0.00047898800946684003 \\
\hline $\mathrm{d}$ & $8.0761221426040233 \mathrm{E}-007$ & $2.4567498334604743 \mathrm{E}-008$ \\
\hline $\mathrm{e}$ & $-9.1633478153210486 \mathrm{E}-011$ & $-8.311832496070721 \mathrm{E}-013$ \\
\hline $\mathrm{f}$ & $6.4674967936309335 \mathrm{E}-015$ & $1.7499832011980812 \mathrm{E}-017$ \\
\hline $\mathrm{g}$ & $-2.5671915421023492 \mathrm{E}-019$ & $-2.0727376423995226 \mathrm{E}-022$ \\
\hline $\mathrm{h}$ & $4.3699398044710495 \mathrm{E}-024$ & $1.0531508226998197 \mathrm{E}-027$ \\
\hline
\end{tabular}

proven to be acceptable and easily implemented. This approach fits a seventh-order polynomial [(9), having 32-digit coefficients] to the "standard" temperature-count transfer function generated by (8) over the temperature range from $-5{ }^{\circ} \mathrm{C}$ to $55^{\circ} \mathrm{C}$ in $2.5^{\circ} \mathrm{C}$ steps, using a nonlinear curve fitting technique [3]. It then takes advantage of the fact that all polynomial evaluation can be reduced to simple addition. This method, while slow, has been adapted here to iteratively compute incremental temperature values above $-4.995{ }^{\circ} \mathrm{C}$ until the temperature value corresponding to the measured count is produced.

$$
f(x)=a+b x+c x^{2}+d x^{3}+e x^{4}+f x^{5}+g x^{6}+h x^{7}
$$

where $f(x)$ represents the temperature and $x$ represents the count value.

The English mathematician and inventor Charles Babbage (1791-1871) faced a similar challenge in 1832 in attempting to mechanically calculate and print error-free high-resolution trigonometric tables for navigational purposes [11]. His "difference engine" (which he only partially built) made use of the finite difference techniques introduced by the English mathematician Henry Briggs in the sixteenth century [4]. The "method of finite differences," developed by Briggs and described in detail by Paige and Koenig [7], can be used to generate a sequence of polynomial values $f\left(x_{0}\right), f\left(x_{0}+s\right)$, $f\left(x_{0}+2 s\right)$, etc., based on the following idea. For a given polynomial $f(x)$ of degree $n$ and an increment $s$, the first difference polynomial $f_{1}(x)=f(x+s)-f(x)$ is of degree $n-1$ or less, the second difference polynomial $f_{2}(x)=f_{1}(x+s)-$ $f_{1}(x)$ is of degree $n-2$ or less ... and, finally, $f_{n}(x)$ must be a constant. Tabulating successive values of $f(x)$ starting with $x=x_{0}$ can be performed in two steps.

1) Initial values for $f\left(x_{0}\right), f_{1}\left(x_{0}\right), \ldots, f_{n}\left(x_{0}\right)$ are derived. For a seventh-order polynomial fitting the general counttemperature curve of Fig. 3, these eight "starting values" are calculated during the sensor design phase, then stored permanently in the sensor's program memory. They are moved to random access memory when temperature is to be calculated from count.

2) Briggs' method requires only $n$ additions to compute each new polynomial value. This addition process is explained in Section III-B below.

\section{A. Calculating Initial Values for a Standard Count-to-Temperature Table}

The eight coefficients $a, b, \ldots h$ of the polynomial in (9) are given in Table I for a low-temperature table ranging between $-5{ }^{\circ} \mathrm{C}$ and $20{ }^{\circ} \mathrm{C}$, and for a high-temperature table ranging between $20{ }^{\circ} \mathrm{C}$ and $50{ }^{\circ} \mathrm{C}$. Two tables are used in practice to shorten the temperature calculation time to less than $10 \mathrm{~s}$, because the calculation must always start at the lowest temperature in the table and count upwards.

The formulas (Table II) needed to calculate the eight starting values of a "Babbage table" for $f(x)$ are simplified in this application because the natural "step" size $s$ of the table is equal to 1 count. The opening value for count $x$ is 2911 at $-5{ }^{\circ} \mathrm{C}$ in the low-temperature table and 9732 at $20{ }^{\circ} \mathrm{C}$ for the hightemperature table.

The derivative values in Table II can be calculated by successive differentiation of the seventh order polynomial as shown in Table III and by using the coefficient values of Table I.

As expected (and fundamental to this method of finite differences) the seventh derivative (the derivative equal to the order of the polynomial) is a constant, equal to $5040 h$, and all higher derivatives are equal to 0 .

Table IV presents the starting values in table form for the low-temperature range. It uses 128-bit (32-digit) numerical precision to maximize the useful range of the function before it starts to diverge unacceptably due to accumulating round-off errors.

\section{B. Calculating Temperature From the Count Value}

Once the eight starting values have been calculated to a precision of 32-digits, they are converted into 16-byte integer numbers of base 256 (Table V) by multiplying them by $256^{14}$. Base 256 is used because microcontrollers can add 8-bit bytes together in a single instruction cycle. Temperatures in the table are represented in units of millidegree Celsius in the range $-10000 \mathrm{~m}^{\circ} \mathrm{C}$ to $+55535 \mathrm{~m}^{\circ} \mathrm{C}$, where values in the top lefthand byte greater than 217 indicate negative temperatures.

The temperature value result (in millidegree Celsius) is the number in Row 0 and Columns 15 and 14 of Table V. In this low-temperature table, the starting temperature is a negative 
TABLE II

Formulae for Calculating the Eight Starting Values FOR A SEVENTH-ORDER POLYNOMIAL WHEN THE TABle STEP Size Is Equal to 1 Count

\begin{tabular}{|c|c|}
\hline$\#$ & Starting values from derivatives \\
\hline 0 & $=f(x)$ \\
\hline 1 & $\begin{array}{l}=f^{\prime}(x)+\frac{1}{2 !} f^{\prime \prime}(x) \\
+\frac{1}{3 !} f^{\prime \prime \prime}(x) \\
+\frac{1}{4 !} f^{\prime \prime \prime \prime}(x) \\
+\frac{1}{5 !} f^{\prime \prime \prime \prime \prime}(x) \\
+\frac{1}{6 !} f^{\prime \prime \prime \prime \prime \prime}(x) \\
+1 / 7 ! f^{\prime \prime \prime \prime \prime ' ~}(x)\end{array}$ \\
\hline 2 & $\begin{array}{l}=2 / 2 ! f^{\prime \prime}(x) \\
+6 / 3 ! f^{\prime \prime \prime}(x) \\
+14 / 4 ! f^{\prime \prime \prime \prime}(x) \\
+30 / 5 ! f^{\prime \prime \prime \prime '(x)} \\
+62 / 6 ! f^{\prime \prime \prime \prime ' '(x)} \\
+126 / 7 ! f^{\prime \prime \prime \prime \prime ' ~}(x)\end{array}$ \\
\hline 3 & $\begin{array}{l}=6 / 3 ! f^{\prime \prime \prime}(x) \\
+36 / 4 ! f^{\prime \prime \prime \prime}(x) \\
+150 / 5 ! f^{\prime \prime \prime \prime '(x)} \\
+540 / 6 ! f^{\prime \prime \prime \prime ' "(x) ~} \\
+1806 / 7 ! f^{\prime \prime \prime \prime ' " '(x)}\end{array}$ \\
\hline 4 & $\begin{array}{l}=24 / 4 ! f^{\prime \prime \prime \prime}(x) \\
+240 / 5 ! f^{\prime \prime \prime \prime '(x)} \\
+1560 / 6 ! f^{\prime \prime \prime \prime ' '(x)} \\
+8400 / 7 ! f^{\prime \prime \prime \prime ' " '(x)}\end{array}$ \\
\hline 5 & $\begin{array}{l}=120 / 5 ! f^{\prime \prime \prime \prime '(x)} \\
+1800 / 6 ! f^{\prime \prime \prime \prime " '(x)} \\
+16800 / 7 ! f^{\prime \prime \prime \prime " '(x) ~}\end{array}$ \\
\hline 6 & $\begin{array}{l}=720 / 6 ! f^{\prime \prime \prime " '(x)} \\
+15120 / 7 ! f^{\prime \prime \prime \prime \prime ' "(x)}\end{array}$ \\
\hline 7 & $=5040 / 7 ! f^{\prime \prime \prime \prime " ' \prime}(x)$ \\
\hline
\end{tabular}

TABLE III

Derivative Values of a Seventh-Order Polynomial

\begin{tabular}{|l|}
\hline$f(x)=a+b x+c x^{2}+d x^{3}+e x^{4}+f x^{5}+g x^{6}+h x^{7}$ \\
\hline$f^{\prime}(x)=b+2 c x+3 d x^{2}+4 e x^{3}+5 f x^{4}+6 g x^{5}+7 h x^{6}$ \\
\hline$f^{\prime \prime}(x)=2 c+6 d x+12 e x^{2}+20 f x^{3}+30 g x^{4}+42 h x^{5}$ \\
\hline$f^{\prime \prime \prime}(x)=6 d+24 e x+60 f x^{2}+120 g x^{3}+210 h x^{4}$ \\
\hline$f^{\prime \prime \prime \prime}(x)=24 e+120 f x+360 g x^{2}+840 h x^{3}$ \\
\hline$f^{\prime \prime \prime \prime \prime}(x)=120 f+720 g x+2520 h x^{2}$ \\
\hline$f^{\prime \prime \prime \prime \prime \prime}(x)=720 g+5040 h x$ \\
\hline$f^{\prime \prime \prime \prime \prime '}(x)=5040 h$ \\
\hline
\end{tabular}

number equal to $\left(\left(236^{*} 256+125\right)-65536\right)=-4995 \mathrm{~m}^{\circ} \mathrm{C}$, or $-4.995{ }^{\circ} \mathrm{C}$. The decimal point effectively sits between columns 14 and 13 but is not shown in Table $\mathrm{V}$ as it is not used in practice. Row 7 is added to row 6 and the result placed in row 6 , then row 6 is added to row 5 and so forth, where
TABLE IV

Real 32-Digit Floating-Point Starting Values For the LOW-TEMPERATURE TABLE

\begin{tabular}{|l|l|}
\hline$\#$ & Value \\
\hline 0 & -4994.5576524617611408021228135316 \\
\hline 1 & 6.5799489534512719099998709556173 \\
\hline 2 & -0.0019181784478796728225256163948577 \\
\hline 3 & $1.0380751101724450654816218174452 \mathrm{e}-6$ \\
\hline 4 & $-6.3257939506981017968734635102 \mathrm{e}-10$ \\
\hline 5 & $3.3135372773156110950382554 \mathrm{e}-13$ \\
\hline 6 & $-1.2072448138646040792372 \mathrm{e}-16$ \\
\hline 7 & $2.202449661453408948 \mathrm{e}-20$ \\
\hline
\end{tabular}

row 7 and column 0 are the least-significant row and column, respectively. Row 7 remains unchanged. The values shown are for a count of 2911. After adding these $8 \times 16$ byte numbers together, the temperature corresponding to a count of 2912 is available in the top left-hand 2 byte of the table.

"Ripple-adding" the table once again produces the next incremental temperature value, and this process continues until the current count value is reached and its temperature value is produced. The further the current temperature is from the starting temperature of the table, the longer the sensor takes to compute a result. This delay would be excessive at warmer water temperatures, therefore, the high-temperature table is used above $20.001{ }^{\circ} \mathrm{C}$ when sensors return a count greater than 9732; this not only affords swifter computation but reduces sensor energy consumption.

\section{CAlibration}

\section{A. Laboratory Calibration of Sensor Strings}

The computation of a "starting table" for each individual sensor manufactured, based on the 32-digit numerical method represented in Tables $\mathrm{I}-\mathrm{V}$, would be onerous indeed if it had to be applied to each and every sensor manufactured and calibrated. Low sensor manufacturing cost is of little value if the cost of calibration is prohibitive.

Thermistor manufacturers address the issue of sensor "interchangeability" by tight control of manufacturing processes to achieve "curve matching" of devices to within $\pm 0.1^{\circ} \mathrm{C}$ over a specified operating range.

With this design, the "curve-matching" of 572 temperature points for each of 26 temperature sensors (Fig. 4) was tested over a $12{ }^{\circ} \mathrm{C}$ to $30{ }^{\circ} \mathrm{C}$ temperature range against the "standard curve" described by (8) and Fig. 3. A simple linear fit matched all sensors to within the $\pm 0.01{ }^{\circ} \mathrm{C}$ specification.

Two-point calibrations of a further 120 sensors in coldwater below $10{ }^{\circ} \mathrm{C}$ and in warm-water above $25{ }^{\circ} \mathrm{C}$ showed that all sensors were matched at a randomly selected midrange temperature around $13{ }^{\circ} \mathrm{C}$ to within $\pm 0.006{ }^{\circ} \mathrm{C}$. This is equivalent to a count variability of \pm 2 counts, where the quantization floor is \pm 1 count ("quantisation" is the smallest discrete signal change that a digital sensor can represent). These count variability figures indicate that the contribution of electrical noise to the measurement error is negligible.

Based on these experimental results, a two-point linear-fit calibration is all that is needed for the sensors in a string to 
TABLE V

This Byte-Integer Starting Table Is all the Sensor Needs to ACCurately Calculate Temperatures From $-4995 \mathrm{~m}^{\circ} \mathrm{C}$ To $20000 \mathrm{~m}^{\circ} \mathrm{C}$, in Steps of One Count From 2911 to 9731. Adding the Rows in the Table From Bottom to Top Produces the Temperature Value in the Top Left-Hand 2 Byte for the NeXt Count Value

\begin{tabular}{|c|c|c|c|c|c|c|c|c|c|c|c|c|c|c|c|c|}
\hline$\#$ & 15 & 14 & 13 & 12 & 11 & 10 & 9 & 8 & 7 & 6 & 5 & 4 & 3 & 2 & 1 & 0 \\
\hline 0 & 236 & 125 & 113 & 61 & 176 & 50 & 51 & 182 & 232 & 57 & 80 & 67 & 95 & 66 & 128 & 25 \\
\hline 1 & 0 & 6 & 148 & 56 & 176 & 234 & 138 & 178 & 143 & 33 & 160 & 182 & 153 & 85 & 85 & 206 \\
\hline 2 & 255 & 255 & 255 & 130 & 91 & 183 & 54 & 109 & 98 & 214 & 91 & 78 & 247 & 65 & 133 & 245 \\
\hline 3 & 0 & 0 & 0 & 0 & 17 & 102 & 108 & 209 & 30 & 175 & 54 & 8 & 120 & 68 & 167 & 22 \\
\hline 4 & 255 & 255 & 255 & 255 & 255 & 253 & 73 & 51 & 49 & 3 & 61 & 80 & 226 & 8 & 128 & 245 \\
\hline 5 & 0 & 0 & 0 & 0 & 0 & 0 & 0 & 93 & 46 & 207 & 85 & 100 & 255 & 20 & 99 & 113 \\
\hline 6 & 255 & 255 & 255 & 255 & 255 & 255 & 255 & 255 & 247 & 78 & 62 & 199 & 87 & 22 & 114 & 193 \\
\hline 7 & 0 & 0 & 0 & 0 & 0 & 0 & 0 & 0 & 0 & 0 & 104 & 1 & 251 & 141 & 18 & 56 \\
\hline
\end{tabular}

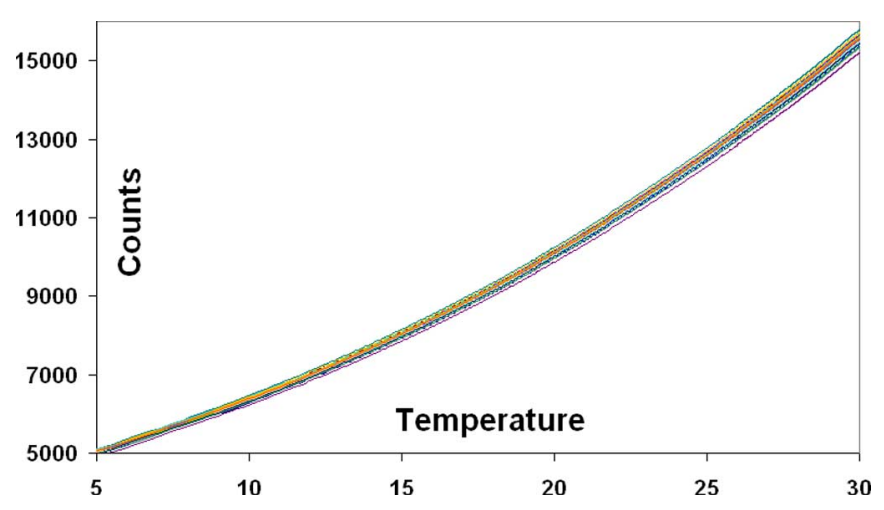

Fig. 4. Counts versus temperature for 26 randomly selected sensors, showing a strong similarity in curve shape. These tests suggested that a simple offset and gain relationship might exist between each sensor and a "standard curve."

be matched to within $\pm 0.01{ }^{\circ} \mathrm{C}$ over the temperature range $0{ }^{\circ} \mathrm{C}$ to $30{ }^{\circ} \mathrm{C}$.

Table $\mathrm{V}$ allows the sensor to dynamically generate a "standard curve" equivalent to a high-precision lookup table containing tens of thousands of points. "Ripple-adding" this table converts the current sensor count to a "standard" temperature $T_{\mathrm{s}}$. This is adjusted by a linear gain and offset term to provide a sensor-specific temperature reading $T$ via (10)

$$
T=\text { offset }+ \text { gain } \times T_{\mathrm{s}} .
$$

Laboratory calibration of a bundled sensor string is undertaken by placing all sensors in a well-stirred insulated water bath alongside a reference sensor of the same type having a traceable calibration uncertainty of $\pm 0.01^{\circ}$. (It is important that the reference sensor has the same thermal mass as the sensors in the string. This prevents temperature phase errors if the temperature in the bath is drifting due to energy injected by the stirring process).

A small software program was developed to allow the calibration computer to read in two calibration temperature values and automatically compute the offset and gain of (10) for each sensor. These two calibration coefficients are written back into nonvolatile memory within each sensor in the string. The coefficients are 16-bit integer values. Extended SDI-12 commands were especially created for the purpose. Each sensor has a unique address in the string.

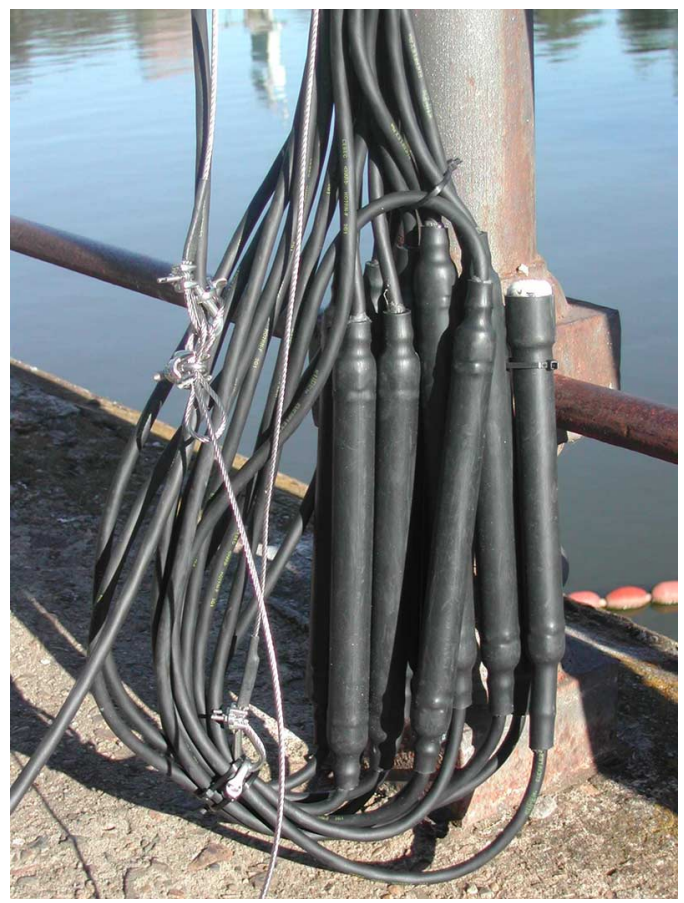

Fig. 5. Sensor string bundled together for two-point in-field calibration at the Torrens Lake in Adelaide South Australia.

\section{B. In-Field Calibration of Sensor Strings}

Long-term drift is of considerable interest to limnologists. The bead-in-glass thermistors used in the sensor are hermetically sealed and are more stable than epoxy-coated or chip thermistors; postcalibration data of 16 sensors after 14 months in the field showed that the worst case calibration drift was $0.009{ }^{\circ} \mathrm{C}$, which is still within the design uncertainty of $\pm 0.01{ }^{\circ} \mathrm{C}$. However, limnologists like to be able to tune strings regularly and inexpensively, and preferably under field conditions. This avoids the large gaps in time-series data sets associated with returning strings to a calibration laboratory.

The "curve-matching" technique of Fig. 4 and (10) allows nonlinear temperature sensors to be calibrated as though they were linear sensors. This opens the possibility for limnologists in the field to recalibrate strings that are exhibiting long-term drift errors.

To test this possibility, 11 sensors in a string were loosely bundled together (Fig. 5) so that they would hang at a common depth, in much the same way as a limnologist would bundle a string for "in-field calibration." Matching of sensors in the 
string was confirmed beforehand to be $\pm 0.006{ }^{\circ} \mathrm{C}$ or better. The sensor bundle was then lowered into the 6-m deep Torrens Lake in Adelaide South Australia, during a period of early-autumn diurnal stratification.

The string was connected to a laptop computer via an SDI12-to-RS232 converter running the calibration program. A small 12-V battery powered the sensor string and the SDI12 converter. The sensor bundle was gently wiggled vertically by $\pm 25 \mathrm{~mm}$ to aid mixing. Temperature readings were logged from all sensors every $2 \mathrm{~min}$ for a period of $1 \mathrm{hr}$ at a depth of $3 \mathrm{~m}$ $\left(21.426{ }^{\circ} \mathrm{C}\right)$ and at a depth of $1 \mathrm{~m}\left(21.994{ }^{\circ} \mathrm{C}\right)$. The temperature gradient over the $2-\mathrm{m}$ water column between the two calibration points was $0.568^{\circ} \mathrm{C}$. Matching throughout the string around a mean temperature fell within an error band of $\pm 0.005{ }^{\circ} \mathrm{C}$ at each depth after $3 / 4 \mathrm{hr}$, even though the temperature at $1-\mathrm{m}$ depth was notably less stable with time than the temperature of the water at 2-m depth.

These matching figures in a natural water body equal those of a well-mixed isothermal calibration tank, suggesting that this "in-field calibration" technique is likely to be practicable even under weakly stratified conditions.

\section{Construction}

\section{A. Sensor Construction}

It is notoriously difficult to waterproof electronics in sensors immersed at depth [6]. It is doubly difficult if they are to be reenterable to make any repairs that may be necessary. Furthermore, one of the most common failure modes of submersible sensors arises from damage to the jacket of the sensor cable-this allows water to wick down inside the cable to fill up and destroy a sensor from within. Low-cost manufacturing techniques were used to achieve waterproofing and serviceability in this design.

The sensors make use of the industry-standard SDI-12 interface bus to connect sensors to data loggers or radios in the buoy on the surface that supports the smart temperature string. The sensors are located at user-selected intervals along a threewire cable like beads on a line. The three conductors in the cable are $+12 \mathrm{~V}$, ground, and the single SDI-12 signal wire. The cable chosen for this application is an Eupen EucrflexPlus H07RN-F 3G1.0 submersible rubber cable. The jacket of this cable is overmoulded onto the three internal conductors that are themselves rubber insulated.

Each sensor is housed in-line within a 205-mm length of lowcost polyvinyl chloride (PVC) pipe having an outside diameter of $21 \mathrm{~mm}$ and an internal diameter of $17.5 \mathrm{~mm}$. The ends of this pipe are internally tapped with a European conduit tap PG11 into which Heyco PG 11 cable glands can be PVC glued in place. These cable glands allow cable entry and exit into the sensor housing that is waterproof to at least $40-\mathrm{m}$ depth. The sensor PCB inside the cylindrical plastic housing is potted inside a reenterable potting compound (Dow-Corning Sylgard 184 Silicone Elastomer). A rubber O-ring is fitted over the rubber cable before it enters the sensor body through the cable gland at each end. Radiform RMW27/8/A4FT heavy-walled adhesive heatshrink is shrunk over the potted sensor assembly,

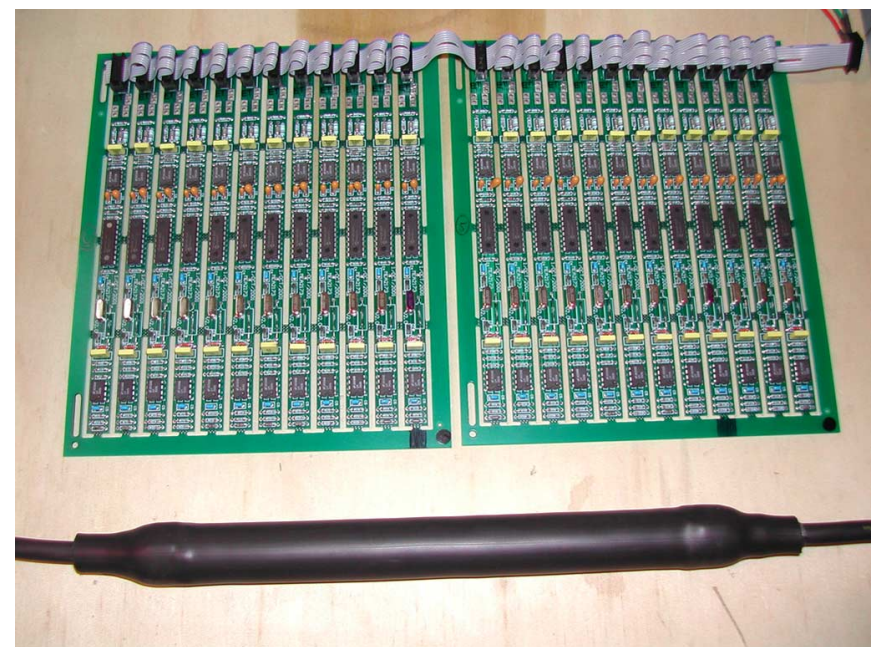

Fig. 6. Twenty-four sensor circuit boards are shown before being broken out from the printed circuit board (PCB) panel form in which they are manufactured. They are shown linked by ribbon cable (top) to power, program, and test them prior to encapsulation. They are then potted inside a threaded PVC tube with a cable-gland and O-ring at each end. A heavy-walled adhesive heatshrink is then shrunk over the whole assembly to form a third level of waterproofing (bottom).

as shown in Fig. 6, to form a low-cost watertight fitting by crushing down the O-rings onto the cable and bonding to all materials via the adhesive.

\section{Sensor Time Constant}

The sensors described in this paper are designed for longterm monitoring of the "fine scale" thermal structure of stratified water columns. The sensor's large thermal time constant of approximately $5 \mathrm{~min}$ (inherent in the mass and materials of the sensor) filters out "micro scale" temperature variations at frequencies above $0.003 \mathrm{~Hz}$.

In contrast, Antenucci and Imberger [2] used high-frequency thermistor chains in Lake Kinneret Israel to observe the internal wave field of this large stratified lake, and showed that highfrequency waves had periods of $\sim 200 \mathrm{~s}$ and caused isotherm displacement of up to $4 \mathrm{~m}$. Lemckert et al. [5] used a freefalling microstructure profiler in association with fast thermistor chains to measure the nature of the turbulence within the benthic boundary layer of the same lake, presenting data showing thermal variations as high as $\pm 2{ }^{\circ} \mathrm{C} \mathrm{m}^{-1}$ around the thermocline. Thorpe [12] used a submarine equipped with a 2.5-m vertical array of 11 thermistors having a time response of $0.02 \mathrm{~s}$, a resolution of $0.001{ }^{\circ} \mathrm{C}$, and a sampling rate of 10 to $30 \mathrm{~Hz}$ to observe the temperature field in Lake Geneva in Switzerland. These authors and others have been concerned with microstructure turbulence and the dissipation of temperature variance as energy is dissipated at smaller and smaller length scales within the water body. While these signals are of interest to scientists studying the mixing processes of lakes and oceans, they represent a source of "thermal noise" to this type of sensor, necessitating a high-thermal mass to prevent signal aliasing. Sampling the thermistor string at minute intervals is consistent with both the energy budget and the storage capabilities of a low-power logged stratification measurement 




Fig. 7. Development of diurnal stratification in White Swan Reservoir in Ballarat, Australia, in May 2003. Note that the natural mixing by dawn on the 28th May is $\pm 0.01{ }^{\circ} \mathrm{C}$ throughout a 16-m water column, and that the data are "consistent" during the development of diurnal stratification later on in the same afternoon. That is, the temperature gradient with depth develops unilaterally; temperature traces do not cross over each other.

system. Climatic parameters, such as wind speed and direction, are often sampled at this same rate to determine wind set-up forces on the lake surface.

\section{DISCUSSION}

A low-cost temperature sensor has been developed that can be configured in submersible "strings" by distributing them along a simple three-wire cable. The sensor has been shown to be capable of delivering simultaneous highly matched temperature measurements to a resolution of a few thousandths of a degree, with a matching of better than $\pm 0.01{ }^{\circ} \mathrm{C}$ and a measurement uncertainty of approximately $\pm 0.05^{\circ} \mathrm{C}$.

Sensor construction techniques have been validated by continuous operation of these sensor strings in the field for periods exceeding 12 months.

A technique for generating a standardized count versus temperature curve has been developed using the method of finite differences. This allows compact code to be used in the sensor; temperature calculations are based only on arithmetic operations. While this is important for code compaction, it also effectively "linearises" the thermistors in the string. This makes possible a simple and quick two-point temperature calibration technique that can be used in the laboratory or for in-field sensor string recalibration to correct for long-term differential drifts between sensors in the strings.

Analysis of data from a sensor string in a reservoir (Fig. 7) showed that a naturally mixing water body could exhibit temperature gradients over a 16-m depth of less than $\pm 0.01{ }^{\circ} \mathrm{C}$, verifying the need for the level of intersensor matching specified and met.

\section{REFERENCES}

[1] Analog Devices, AD652 Monolithic Synchronous Voltage-to-Frequency Converter. Data Converter Reference Manual, vol. 2, pp. 3-27, 1992.

[2] J. P. Antennucci and J. Imberger, "On internal waves near the highfrequency limit in an enclosed basin," J. Geophys. Res., vol. 106, no. C10, pp. 22 465-22 474, Oct. 2001.

[3] J. E. Dennis, D. M. Gay, and R. E Welsch, "An adaptive non-linear leastsquares algorithm,” ACM Trans. Math. Softw., vol. 7, no. 3, pp. 348-368, Sep. 1981.

[4] H. H. Goldstine, A History of Numerical Analysis. New York: SpringerVerlag, 1977.

[5] C. Lemckert, J. Antenucci, A. Saggio, and J. Imberger, "Physical properties of turbulent benthic boundary layers generated by internal waves," ASCE J. Hydraul. Eng., vol. 130, no. 1, pp. 58-69, Jan. 2004.

[6] B. Miaschi and K. E. Rasmussen, Neoprene Vulcanization for Waterproofing of Underwater Cable Systems, Oct. 1974. [Online]. Available: http:// adsabs.harvard.edu/abs/1974STIN...7526276M

[7] R. Paige and S. Koenig, "Finite differencing of computable expressions," ACM Trans. Program. Lang. Syst., vol. 4, no. 3, pp. 402-454, Jul. 1982.

[8] J. C. Rutherford, J. B. MacCaskill, and B. L. Williams, "Natural water temperature variations in the lower Waikato River New Zealand," N.Z. J. Mar. Freshw. Res., vol. 27, pp. 71-85, 1993.

[9] SDI-12 Sensor Interface Bus. [Online]. Available: www.sdi-12.org

[10] J. S. Steinhart and S. R. Hart, "Calibration curves for thermistors," Deep Sea Res., vol. 15, p. 497, 1968

[11] D. Swade, The Cogwheel Brain: Charles Babbage and the Quest to Build the First Computer. London, U.K.: Little, Brown \& Co., 2000.

[12] S. A. Thorpe, "Observations of the thermal structure of a lake using a submarine," Limnol. Oceanogr., vol. 44, no. 6, pp. 1575-1582, 1999.

[13] "YSI precision thermistor thermometry: Application Note TD002," in Proc. Meas. Sci. Conf. Tutor., J. M. Zurbuchan, Ed, 1993/2000.

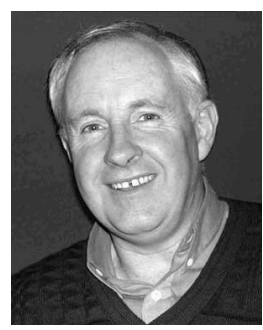

Andrew J. Skinner received the Bachelor of Technology degree in electronic engineering and the Master of Electronic Engineering degree, both from South Australian Institute of Technology, Australia, in 1975 and 1987, respectively.

He worked in Australia, Papua New Guinea, and Canada as a Nucleonic Instrumentation Design Engineer in the mineral processing industry. He returned to Australia in late 1982 to specialize in data logging applications and environmental measurement systems, founding Measurement Engineering Australia in 1984.

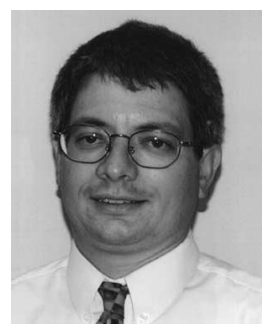

Martin F. Lambert received the Ph.D. degree from University of Newcastle, Australia.

He joined the University of Adelaide in 1995 after completing his Ph.D. He is currently an Associate Professor in the school of Civil and Environmental Engineering. He research areas include river hydraulics, pipeline condition assessment, and stochastic hydrology. 